\title{
Informatics: Emerging Concepts and Issues
}

Lynn M. Nagle, RN, PhD

This new column demonstrates the wisdom and foresight of CJNL's editor for agreeing to include regular commentary on issues of "eHealth" and informatics as they pertain to nurses. In my work as the Senior Nursing Advisor to Canada Health Infoway on the "End-User Engagement Strategy," I have found a need to clarify the many acronyms and concepts applied to this work and address the persistent confusion related to them. Without a shared understanding of core elements, the notion of "engaging nurses" will likely continue to be fraught with frustration and miscommunication. In this first column, I offer some points of clarification of the "alphabet soup." In future columns, I will attempt to address other terminally confounded issues and topics of relevance to nurses.

\section{EHR versus EMR versus EPR}

There are some fundamental distinctions among the terms electronic medical record (EMR), electronic health record (EHR) and electronic patient record (EPR). In many instances, these terms are treated as synonymous and applicable to any comprehensive, longitudinal record with no specified user community, functionality or delineated-scope information capture. However, the distinctions among these terms can be clarified on the basis of access, scope of informational components and custodianship.

In general, EMRs refer to electronic records maintained within a clinic, private practitioner's office, Family Health team and so on. EPRs, on the other hand, 
generally refer to electronic records managed by healthcare organizations. In most cases, EMRs and EPRs are a computerized incarnation of their paper predecessors. Typically, the stewardship of EMRs and EPRs is held by the organizational entity and access is limited to authorized users within the "circle of care." Nevertheless, it is important to note that data may be exchanged among multiple entities, with opportunities for shared infrastructure and several instances of the same vendor solution being used within a single organization.

In contrast, the EHR can be viewed as a comprehensive record for a specific individual, one that incorporates selected information from every healthcare encounter. There is no paper precursor of an EHR; indeed, it would be inordinately challenging, if at all possible, to aggregate all the health information that exists for the average Canadian. Ultimately, an EHR will be constituted by data drawn from every EMR and EPR that is maintained for an individual. The EHR also draws from other point-of-service systems, such as pharmacies and diagnostic testing centres. The EHR is not, however, a mega-repository of every single data element generated by every provider delivering care to every citizen. Within the context of an EHR, health information is owned by the individual, and access to it is gained by consent of the individual who owns it; but the physical record will be under the stewardship of a jurisdiction. Theoretically, an EHR will be accessible to any authorized clinical provider from any point of care within a jurisdiction, and from authorized providers outside the jurisdiction.

Currently, Infoway's blueprint considers EMRs and EPRs to be "point-of-service applications" connected to the EHR, and these connections are controlled by datasharing policies and agreements. Infoway also considers that the EHR will hold the "key health history" of Canadians, the scope of which currently includes information related to labs, diagnostic imaging, drugs, allergies, past medical history and current diagnoses. The final embodiment of the information holdings of the EHR is yet to be delineated.

Another emerging focus of healthcare provider organizations is the design and deployment of "patient portals." Using the Internet, these windows or views of one's personal health information typically facilitate the empowerment of individuals and families by providing access to diagnostic results, health information and education, and patient support communities and forums. Although primarily passive tools for accessing information, portals are evolving to support telemonitoring and the exchange of data between individuals in the home and healthcare providers. In sum, portal technology can reduce the need for in-person visits and monitoring: connectivity and communication between patients and families and their health professionals can occur remotely in real time. 
Just to muddy the waters, consider the emergence of applications to support the management of "personal health records," or PHRs. A number of Internet tools are now available to support the creation of a PHR to self-manage discrete aspects of personal health information related to specific disease entities (e.g., diabetes). In addition, tools are starting to be used to manage comprehensive information pertaining to one's health history, information that can be formatted for sharing with healthcare providers and perhaps kept on one's person in the event of an emergency. In future, these tools may also provide links into EMRs, EPRs and/or the EHR; permutations as yet unimagined may evolve in the future. Stay tuned for more to come on the evolving PHR.

In the meantime, I think we need to continue laying the foundations of the EMRs and EPRs to support the evolution of the EHR. There is much to learn on this journey. As the vision is embraced, shared understandings will continue to emerge until such time as the need to articulate distinctions will be moot.

\section{ACEN Scholarship}

The Academy of Canadian Executive Nurses is pleased to offer a scholarship in the amount of $\$ 2,500$ to be awarded annually to a nurse pursuing graduate studies in nursing, at the Master's or Doctoral level. This scholarship is designed to provide support for

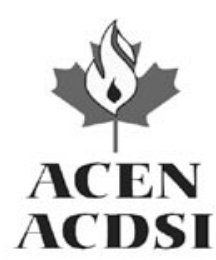
a candidate preparing her/himself for nursing leadership positions within Canada.

\section{Criteria for Eligibility and Selection}

1. Demonstrated interest in and aptitude for nursing leadership based on prior nursing experience.

2. Demonstrated commitment to the nursing profession based upon interest and involvement in associations or groups to promote high standards of client care or projects to promote "best practices" in client care.

3. Confirmed admission to a graduate program.

4. If already registered in a program with partial completion of studies, provision of evidence of sound performance in the completed course work within that program.

5. Demonstrated interest in, and commitment to advancing nursing leadership in Canada.

For application and selection information please visit ACEN's website at: http://www.acen.ca.

\section{Deadline}

Completed applications must be received no later than April 30, 2007. 